\title{
TWO METHODS OF INTEGRATING MONGE-AMPÈRE'S EQUATIONS. II
}

\author{
BY \\ MICHIHIKO MATSUDA
}

\begin{abstract}
Generalizing the notion of an integrable system given in the previous note [2], we shall define an integrable system of higher order, and obtain the following results:

1. A linear hyperbolic equation is solved by integrable systems of order $n$ if and only if its $(n+1)$ th Laplace invariant $H_{n}$ vanishes.

2. An equation of Laplace type is solved by integrable systems of the second order if and only if the transformed equation by the associated Imschenetsky transformation is solved by integrable systems of the first order.
\end{abstract}

0. Introduction. Let us solve a linear hyperbolic equation

$$
s+a p+b q+c z=0
$$

by Laplace's method, where $a, b, c$ are functions of $x, y$, and $p=\partial z / \partial x, q=\partial z / \partial y$, $s=\partial^{2} z / \partial x \partial y$. The integration is reduced to that of ordinary differential equations if the first Laplace invariant $H_{0}=\partial a / \partial x+a b-c$ vanishes; for in this case equation (1) has the form

$$
(\partial / \partial x)(\partial z / \partial y+a z)+b(\partial z / \partial y+a z)=0
$$

Suppose that the first invariant $H_{0}$ does not vanish. Then we can transform equation (1) by the Laplace transformation $z_{1}=q+a z, H_{0} z=\partial z_{1} / \partial x+b z_{1}$ to the equation

$$
(\partial / \partial x)\left(\partial z_{1} / \partial y+a_{1} z_{1}\right)+b\left(\partial z_{1} / \partial y+a_{1} z_{1}\right)-H_{1} z_{1}=0,
$$

where

$$
H_{1}=\partial a_{1} / \partial x-\partial b / \partial y+H_{0}, \quad a_{1}=a-\partial\left(\log H_{0}\right) / \partial y .
$$

The $(n+1)$ th Laplace invariant $H_{n}$ is defined inductively by $H_{n}=\partial a_{n} / \partial x-\partial b / \partial y$ $+H_{n-1}$, unless $H_{n-1}=0$, where $a_{n}=a_{n-1}-\partial\left(\log H_{n-1}\right) / \partial y$. The given equation (1) is transformed to the equation

$$
(\partial / \partial x)\left(\partial z_{n} / \partial y+a_{n} z_{n}\right)+b\left(\partial z_{n} / \partial y+a_{n} z_{n}\right)-H_{n} z_{n}=0
$$

Received by the editors March 1, 1971.

AMS 1970 subject classifications. Primary 35A22, 35A30, 35G20.

Key words and phrases. Monge-Ampère's equation, Laplace invariant, integrable system, Bäcklund transformation, Imschenetsky transformation.

Copyright (C) 1972, American Mathematical Society 
by successive application of the Laplace transformation. Hence, if the $(n+1)$ th invariant $H_{n}$ vanishes, then we can solve (1) by integrating ordinary differential equations.

Here we shall discuss the problem from a different point of view. It is Cauchy who first tried to solve the initial value problem of a partial differential equation by integrating ordinary differential equations. He proved that the initial value problem of an equation of the first order $V(x, y, z, p, q)=0$ is solved by integrating the Lagrange-Charpit system

$$
\frac{d x}{\partial V / \partial p}=\frac{d y}{\partial V / \partial q}=\frac{d z}{p \partial V / \partial p+q \partial V / \partial q}=\frac{-d p}{d V / d x}=\frac{-d q}{d V / d y}
$$

Here $d / d x=\partial / \partial x+p(\partial / \partial z), d / d y=\partial / \partial y+q(\partial / \partial z)$. The Cauchy problem of MongeAmpère's equation is solved by integrating the Lagrange-Charpit system of an intermediate integral of the first order if and only if Monge's method of integration is applied to the given equation with success. Generalizing the Lagrange-Charpit system, we defined an integrable system in [2]. Consider a system of ordinary differential equations

$$
\frac{d x}{A}=\frac{d y}{B}=\frac{d z}{A p+B q}=\frac{d p}{C}=\frac{d q}{D}
$$

in the space of $(x, y, z, p, q)$, where $A, B, C, D$ are functions of $x, y, z, p, q$. Then it is called an integrable system if, for any initial curve satisfying $d z-p d x-q d y=0$ and $A d p+B d q-C d x-D d y=0$, the integral surface of the system satisfies $d z-p d x-q d y=0$. We showed that the Cauchy problem of the linear hyperbolic equation (1) is solved by integrable systems if and only if $H_{1}=0$.

Expanding this notion of an integrable system, we shall define here an integrable system of higher order to solve the Cauchy problem of an equation of the form

$$
s+f(x, y, z, p, q)=0
$$

in the space of $\left(x, y, z, p, q_{1}, \ldots, q_{n}\right)$ containing the derivatives of higher order $q_{i}=\partial^{i} z / \partial y^{i}$. In this space we shall consider a system of ordinary differential equations

$$
\begin{aligned}
& \frac{d x}{d y}=0, \quad \frac{d z}{d y}=q_{1}, \quad \frac{d p}{d y}=-f, \\
& \frac{d q_{i}}{d y}=q_{i+1}, \quad 1 \leqq i<n, \quad \frac{d q_{n}}{d y}=u
\end{aligned}
$$

and differential forms

$$
\begin{aligned}
\omega_{0} & =d z-p d x-q d y, \\
\omega_{i} & =d q_{i}+f_{i-1} d x-q_{i+1} d y, \quad 1 \leqq i<n, \\
\omega & =d q_{n}+f_{n-1} d x-u d y,
\end{aligned}
$$


where $u$ is a function of $\left(x, y, z, p, q_{1}, \ldots, q_{n}\right)$ to be determined later and $f_{i}$ is the function defined inductively by

$$
\begin{aligned}
f_{i+1} & =\frac{\partial f_{i}}{\partial y}+\frac{\partial f_{i}}{\partial z} q_{1}-\frac{\partial f_{i}}{\partial p} f+\sum_{j=1}^{i+1} \frac{\partial f_{i}}{\partial q_{j}} q_{j+1}, \quad 0 \leqq i<n-1, \\
f_{0} & =f .
\end{aligned}
$$

We shall say that the system (3) is integrable, if for any initial curve

$$
y=y_{0}(x), \quad z=z_{0}(x), \quad p=p_{0}(x), \quad q_{i}=q_{i 0}(x), \quad 1 \leqq i \leqq n,
$$

satisfying

$$
\omega_{0}=\omega_{1}=\cdots=\omega_{n-1}=\omega=0,
$$

the integral surface of the system (3)

$$
z=\varphi(x, y), \quad p=\psi(x, y), \quad q_{i}=\varphi_{i}(x, y), \quad 1 \leqq i \leqq n,
$$

under the initial condition (4) satisfies (5). In this sense the integrable system defined in [2] is the integrable system of the first order. If the system (3) is integrable, then the integral surface (6) of (3) is a solution of the given equation (2) for we have, from (5),

$$
\begin{aligned}
& \frac{\partial \varphi}{\partial x}=\psi(x, y), \quad \frac{\partial \varphi}{\partial y}=\varphi_{1}(x, y), \quad \frac{\partial \varphi_{i}}{\partial y}=\varphi_{i+1}, \quad 1 \leqq i<n, \\
& \frac{\partial \varphi_{i}}{\partial x}=-f_{i-1}\left(x, y, \varphi, \psi, \varphi_{1}, \ldots, \varphi_{i}\right), \quad 1 \leqq i \leqq n,
\end{aligned}
$$

and hence

$$
\frac{\partial \psi}{\partial y}=\frac{\partial^{2} \varphi}{\partial x \partial y}=\frac{\partial \varphi_{1}}{\partial x}, \quad \frac{\partial}{\partial y} \frac{\partial \varphi_{i-1}}{\partial x}=-f_{i-1}=\frac{\partial \varphi_{i}}{\partial x}, \quad 1 \leqq i \leqq n .
$$

We shall say that the equation (2) is solved by integrable systems of order $n$, if for any initial curve (4) satisfying $\omega_{0}=\omega_{1}=\cdots=\omega_{n-1}=0$, we can find such a function $u$ that satisfies $\omega=0$ along (4) and makes the system (3) integrable. Apply this method of integration to the linear hyperbolic equation (1). Then we obtain the following theorem:

The equation (1) is solved by integrable systems of order $n$ if and only if its $(n+1)$ th Laplace invariant $H_{n}$ vanishes.

As the next problem let us try to generalize this theorem. Presenting an example of Bäcklund transformations, Imschenetsky generalized the Laplace transformation as follows (see [1]): Consider a transformation

$$
x_{1}=x, \quad y_{1}=y, \quad z_{1}=h(x, y, z, q), \quad p_{1}=k(x, y, z, q)
$$

satisfying $\partial h / \partial q \neq 0, \partial(h, k) / \partial(z, q) \neq 0$. Suppose that a surface $z=\varphi(x, y)$ is a solution of the equation

$$
\frac{\partial h}{\partial q} s+\frac{\partial h}{\partial z} p+\frac{\partial h}{\partial x}-k=0 .
$$


Then the derivative of the function

$$
z_{1}=h\left(x_{1}, y_{1}, \varphi\left(x_{1}, y_{1}\right), \varphi_{y}\left(x_{1}, y_{1}\right)\right)
$$

with respect to $x_{1}$ is $k\left(x_{1}, y_{1}, \varphi, \varphi_{y}\right)$, since

$$
\frac{\partial z_{1}}{\partial x_{1}}=\frac{\partial h}{\partial x}+\frac{\partial h}{\partial z} \varphi_{x}+\frac{\partial h}{\partial q} \varphi_{x y}=k
$$

The surface (9) is a solution of the equation

$$
\frac{\partial h}{\partial q} s_{1}=\frac{\partial k}{\partial q} q_{1}+\frac{d k}{d y} \frac{\partial h}{\partial q}-\frac{d h}{d y} \frac{\partial k}{\partial q}
$$

where we replace $x, y, z, q$ by their values obtained from (7). The equation (10) is derived from

$$
s_{1}=\frac{d k}{d y}+\frac{\partial k}{\partial q} \varphi_{y y}, \quad q_{1}=\frac{d h}{d y}+\frac{\partial h}{\partial q} \varphi_{y y}
$$

Thus the Imschenetsky transformation (7) transforms the equation (8) to the equation (10). We shall say that an equation of the form

$$
s+M(x, y, s, q) p+N(x, y, z, q)=0
$$

is of Imschenetsky type, if its first invariant $H=\partial M / \partial x-N(\partial M / \partial q)-\partial N / \partial z$ $+M(\partial N / \partial q)$ does not vanish. This is a necessary and sufficient condition that an Imschenetsky transformation be applied to the equation (11). Also the condition $H=0$ is a necessary and sufficient one that the equation (11) be Monge-integrable. A Monge-integrable equation is by definition an equation whose Cauchy problem is solved by integrating Lagrange-Charpit systems (see [2]). The inverse transformation of the Imschenetsky transformation (7) is given by solving (7) conversely with respect to $x, y, z, q$. An equation of the form

$$
s_{1}+M_{1}\left(x_{1}, y_{1}, z_{1}, p_{1}\right) q_{1}+N_{1}\left(x_{1}, y_{1}, z_{1}, p_{1}\right)=0
$$

is the transformed equation of an Imschenetsky transformation if and only if $K_{1}=\partial M_{1} / \partial y_{1}-N_{1}\left(\partial M_{1} / \partial p_{1}\right)-\partial N_{1} / \partial z_{1}+M_{1}\left(\partial N_{1} / \partial p_{1}\right)$ does not vanish. In this case we have the identity $H=K_{1}$.

In the previous note [2] we defined an equation of Laplace type as an equation of Imschenetsky type whose transformed equation has the form

$$
s_{1}-\left(\gamma p_{1}+\delta\right) q_{1}-\left(\alpha p_{1}+\beta\right)=0,
$$

where $\alpha, \beta, \gamma, \delta$ are functions of $x_{1}, y_{1}, z_{1}$. The equation (11) of Imschenetsky type is of Laplace type if the transformed equation is Monge-integrable, for an equation of the form (2) is not Monge-integrable, unless the function $f$ is linear with respect to $p$. In [2] we showed that an equation of Laplace type is solved by integrable systems of the first order if and only if the transformed equation is Monge-integrable. In this note we shall obtain a necessary and sufficient condition which the 
coefficients $M$ and $N$ should satisfy in order that the equation (11) of Imschenetsky type may be of Laplace type. And we shall show that the equation (11) of Imschenetsky type is of Laplace type if it is solved by integrable systems of the first order. The following theorem will be proved:

An equation of Laplace type is solved by integrable systems of the second order if and only if the transformed equation is solved by integrable systems of the first order.

If an equation of Laplace type is solved by integrable systems of the first order, it is solved by integrable systems of the second order. Hence an equation of Laplace type is transformed to a Monge-integrable equation by applying once or twice the associated Imschenetsky transformation if and only if it is solved by integrable systems of the second order.

REMARK 1. Equation (2) has the two characteristics $d x=0, d p+f d y=0$ and $d y=0, d q+f d x=0$. The same argument as above can be made with respect to the other characteristic.

RemarK 2. By Professor G. L. Lamb, Jr. in Physics the author was informed of Clairin's work [1]. The Bäcklund transformation of Laplace type defined in [2] is the same as the Imschenetsky transformation applied to an equation of Laplace type.

1. Laplace invariants. Let us define an integrable system of order $n$ by the following:

Definition. The system (3) is integrable if the function $u$ satisfies the system of the equations

$$
\begin{gathered}
\frac{\partial u}{\partial p}=0 \\
\frac{\partial u}{\partial x}+p \frac{\partial u}{\partial z}-f \frac{\partial u}{\partial q}-\sum_{i=1}^{n-1} f_{i} \frac{\partial u}{\partial q_{i+1}}+\frac{\partial f_{n-1}}{\partial y}+q_{1} \frac{\partial f_{n-1}}{\partial z}-f \frac{\partial f_{n-1}}{\partial p} \\
+\sum_{i=1}^{n-1} q_{i+1} \frac{\partial f_{n-1}}{\partial q_{i}}+\frac{\partial f}{\partial q} u=0 .
\end{gathered}
$$

In the proof of the following proposition, we shall see that this definition is equivalent to the one given in $\$ 0$.

Proposition 1. Suppose that the system (3) is integrable. Then for any initial curve (4) satisfying (5), the integral surface (6) of (3) under the initial condition (4) satisfies (5).

Proof. On the integral surface (6) we have

$$
\begin{aligned}
& \partial \varphi / \partial y-\varphi_{1}=0, \quad \partial \varphi_{i} / \partial y-\varphi_{i+1}=0, \quad 1 \leqq i<n, \\
& \partial \varphi_{n} / \partial y-u_{0}=0, \quad \partial \psi / \partial y+f=0,
\end{aligned}
$$

where

$$
u_{0}(x, y)=u\left(x, y, \varphi, \psi, \varphi_{1}, \ldots, \varphi_{n}\right)
$$


Let $I$ be the left-hand member of the second equation in (12), and put

$$
v_{0}(x, y)=\partial \varphi / \partial x-\psi, \quad v_{i}(x, y)=\partial \varphi_{i} / \partial x+g_{i-1}, \quad 1 \leqq i \leqq n,
$$

where

$$
g_{i-1}(x, y)=f_{i-1}\left(x, y, \varphi, \psi, \varphi_{1}, \ldots, \varphi_{i}\right), \quad 1 \leqq i \leqq n .
$$

Then we get $\partial v_{i} / \partial y=v_{i+1}, 0 \leqq i<n$, and

$$
\frac{\partial v_{n}}{\partial y}=\frac{\partial u}{\partial z} v_{0}+\sum_{i=1}^{n} \frac{\partial u}{\partial q_{i}} v_{i}+\frac{\partial u}{\partial p} \frac{\partial \psi}{\partial x}+I .
$$

Here we replace $z, p, q_{1}, \ldots, q_{n}$ in $\partial u / \partial z, \partial u / \partial p, \partial u / \partial q_{i}, 1 \leqq i \leqq n$, and $I$ by $\varphi, \psi$, $\varphi_{1}, \ldots, \varphi_{n}$ respectively. By the assumption that the given system (3) is integrable, the right-hand member of the last equation for $\partial v_{n} / \partial y$ is linear with respect to $v_{i}$, $0 \leqq i \leqq n$. Since $v_{i}=0(0 \leqq i \leqq n)$ along the initial curve (4), we have $v_{i}=0$ on (6), $0 \leqq i \leqq n$.

Hence equation (2) is solved by integrable systems of order $n$ if and only if the system (12) with the unknown function $u$ has its rank greater than zero (see [2]).

Apply this method of integration to the linear hyperbolic equation (1), where $f=a p+b q+c z$. Let us define $A_{i}, B_{i j}, C_{i}(i, j \geqq 0)$ inductively by

$$
\begin{array}{rlrl}
A_{i} & =\partial A_{i-1} / \partial y-a A_{i-1}, & & i \geqq 1, \\
B_{i j} & =\partial B_{i-1, j} / \partial y+B_{i-1, j-1}, & & i, j \geqq 1, \\
C_{i} & =\partial C_{i-1} / \partial y-c A_{i-1}, & i \geqq 1, & \\
A_{0} & =a, \quad C_{0}=c, \quad B_{i 0}=C_{i}-b A_{i}, \quad B_{01}=b, \\
B_{0 j} & =0, \quad j>1 .
\end{array}
$$

Then we get $B_{i, i+1}=b, B_{i j}=0, j>i+1$, and

$$
f_{i}=A_{i} p+\sum_{j=1}^{i+1} B_{i j} q_{j}+C_{i} z, \quad i \geqq 0 .
$$

The system (12) is equivalent to the system of the equations

$$
\begin{aligned}
Z_{n} u+A_{n} & =0, \\
X_{n} u+b u+\sum_{j=1}^{n} B_{n j} q_{j}+C_{n} z & =0
\end{aligned}
$$

with independent variables $x, y, z, q_{1}, \ldots, q_{n}$, where

$$
\begin{aligned}
& Z_{n}=\frac{\partial}{\partial z}-\sum_{i=0}^{n-1} A_{i} \frac{\partial}{\partial q_{i+1}}, \\
& X_{n}=\frac{\partial}{\partial x}-\sum_{i=0}^{n-1}\left(\sum_{j=1}^{i+1} B_{i j} q_{j}+C_{i} z\right) \frac{\partial}{\partial q_{i+1}} .
\end{aligned}
$$

Suppose that the $n$th invariant $H_{n-1}$ does not vanish and hence the $(n+1)$ th invariant $H_{n}$ can be defined. Let us define $A_{m i}(0 \leqq m \leqq n,-1 \leqq i)$ inductively by 


$$
\begin{gathered}
H_{m} A_{m+1, i}=\frac{\partial A_{m i}}{\partial x}+\sum_{j=0}^{i} A_{m, j-1} B_{i j}, \quad m, i \geqq 0, \\
A_{0,-1}=-1, \quad A_{0 i}=A_{i}, \quad i \geqq 0, \quad A_{m,-1}=0, \quad m>0,
\end{gathered}
$$

and differential operators $Y_{m n}(1 \leqq m \leqq n)$ by

$$
Y_{m n}=\sum_{j=1}^{n} A_{m, j-1} \frac{\partial}{\partial q_{j}} \text {. }
$$

Then we have $\left[Z_{n}, X_{n}\right]=H_{0} Y_{1 n},\left[X_{n}, Y_{m n}\right]=H_{m} Y_{m+1, n}+b Y_{m n}, m<n$. As the compatibility condition between the equations (13) and (14) we get

$$
Y_{1 n} u-A_{1 n}=0 \text {. }
$$

From this equation we derive $Y_{m n} u-A_{m n}=0,2 \leqq m \leqq n$, successively as the compatibility condition between (14) and $Y_{m-1, n} u-A_{m-1, n}=0$. The compatibility condition between (14) and the last equation $Y_{n n} u-A_{n n}=0$ is

$$
\sum_{j=0}^{n-1}\left(\frac{\partial A_{n j}}{\partial x}+\sum_{i=0}^{j} A_{n, i-1} B_{j i}\right) \frac{\partial u}{\partial q_{j+1}}=\frac{\partial A_{n n}}{\partial x}+\sum_{i=0}^{n} A_{n, i-1} B_{n i} .
$$

LEMMA. For each $m(1 \leqq m \leqq n)$ we have the following identities:

(i) $A_{m, m-1}=(-1)^{m-1}$;

(ii) $A_{m i}=0,0 \leqq i<m-1$;

(iii) $\partial A_{m m} / \partial x+(-1)^{m-1} B_{m m}=(-1)^{m} H_{m}$;

(iv) $A_{m i}=\partial A_{m, i-1} / \partial y-a_{m} A_{m, i-1}-A_{m-1, i-1}, i \geqq 1$.

Proof. As the first step let us prove these identities for $m=1$. By the definition we get $H_{0} A_{10}=\partial A_{00} / \partial x+A_{0,-1} B_{00}=\partial a / \partial x-(c-b a)=H_{0}$, and

$$
\begin{aligned}
H_{0} A_{1 i}= & \frac{\partial A_{0 i}}{\partial x}+\sum_{j=0}^{i} A_{0, j-1} B_{i j} \\
= & \frac{\partial}{\partial x}\left(\frac{\partial A_{i-1}}{\partial y}-a A_{i-1}\right)+b\left(\frac{\partial A_{i-1}}{\partial y}-a A_{i-1}\right)-\left(\frac{\partial C_{i-1}}{\partial y}-c A_{i-1}\right) \\
& +\sum_{j=1}^{i} A_{j-1} \frac{\partial B_{i-1, j}}{\partial y}+a B_{i-1,0}+\sum_{j=2}^{i}\left(\frac{\partial A_{j-2}}{\partial y}-a A_{j-2}\right) B_{i-1, j-1} \\
= & \frac{\partial}{\partial y}\left(\frac{\partial A_{i-1}}{\partial x}+b A_{i-1}-C_{i-1}+\sum_{j=1}^{i-1} A_{j-1} B_{i-1, j}\right) \\
& -a\left(\frac{\partial A_{i-1}}{\partial x}+b A_{i-1}-C_{i-1}+\sum_{j=1}^{i-1} A_{j-1} B_{i-1, j}\right)-(\partial a / \partial x+a b-c) A_{i-1} \\
= & (\partial / \partial y)\left(H_{0} A_{1, i-1}\right)-a H_{0} A_{1, i-1}-H_{0} A_{0, i-1} .
\end{aligned}
$$

These identities give (i) and (iv), since $a_{1}=a-\partial\left(\log H_{0}\right) / \partial y$. Hence we have

$$
\begin{aligned}
\frac{\partial A_{11}}{\partial x}+B_{11} & =\frac{\partial}{\partial x}\left(\frac{\partial A_{10}}{\partial y}-a_{1} A_{10}-A_{00}\right)+\frac{\partial B_{01}}{\partial y}+B_{00} \\
& =-\frac{\partial}{\partial x}\left(a_{1}+a\right)+\frac{\partial b}{\partial y}+c-a b=-\frac{\partial a_{1}}{\partial x}+\frac{\partial b}{\partial y}-H_{0}=-H_{1} .
\end{aligned}
$$


As the second step, let us suppose that the identities (i)-(iv) hold for each of $1,2, \ldots, m$, and prove them for $m+1$. By (i)-(iii) we get

$$
\begin{aligned}
& H_{m} A_{m+1, i}=\frac{\partial A_{m i}}{\partial x}+\sum_{j=0}^{i} A_{m, j-1} B_{i j}=0, \quad 0 \leqq i<m, \\
& H_{m} A_{m+1, m}=\frac{\partial A_{m m}}{\partial x}+\sum_{j=0}^{m} A_{m, j-1} B_{m j}=(-1)^{m} H_{m} .
\end{aligned}
$$

By the identities (iv) and $H_{m}-H_{m-1}=\partial a_{m} / \partial x-\partial b / \partial y$, we obtain

$$
\begin{aligned}
H_{m} A_{m+1, i}= & \frac{\partial A_{m i}}{\partial x}+\sum_{j=m}^{i} A_{m, j-1} B_{i j} \\
= & \frac{\partial}{\partial x}\left(\frac{\partial A_{m, i-1}}{\partial y}-a_{m} A_{m, i-1}-A_{m-1, i-1}\right)+\sum_{j=m}^{i} A_{m, j-1} \frac{\partial B_{i-1, j}}{\partial y} \\
& +\sum_{j=m}^{i}\left(\frac{\partial A_{m, j-2}}{\partial y}-a_{m} A_{m, j-2}-A_{m-1, j-2}\right) B_{i-1, j-1} \\
= & \frac{\partial}{\partial y}\left(\frac{\partial A_{m, i-1}}{\partial x}+\sum_{j=m}^{i-1} A_{m, j-1} B_{i-1, j}\right)-a_{m}\left(\frac{\partial A_{m, i-1}}{\partial x}+\sum_{j=m}^{i-1} A_{m, j-1} B_{i-1, j}\right) \\
& -\left(\frac{\partial A_{m-1, i-1}}{\partial x}+\sum_{j=m-1}^{i-1} A_{m-1, j-1} B_{i-1, j}\right)-\left(\frac{\partial a_{m}}{\partial x}-\frac{\partial B_{i-1, i}}{\partial y}\right) A_{m, i-1} \\
= & (\partial / \partial y)\left(H_{m} A_{m+1, i-1}\right)-a_{m}\left(H_{m} A_{m+1, i-1}\right)-H_{m} A_{m, i-1} .
\end{aligned}
$$

This gives (iv) for $m+1$, since $a_{m+1}=a_{m}-\partial\left(\log H_{m}\right) / \partial y$. Hence we get

$$
\begin{aligned}
(\partial / \partial x) A_{m+1, m+1}+( & -1)^{m} B_{m+1, m+1} \\
& =(-1)^{m+1}\left\{\frac{\partial a_{m+1}}{\partial x}-(-1)^{m+1} \frac{\partial A_{m m}}{\partial x}-\frac{\partial B_{m, m+1}}{\partial y}-B_{m m}\right\} \\
& =(-1)^{m+1}\left(\partial a_{m+1} / \partial x-\partial b / \partial y+H_{m}\right)=(-1)^{m+1} H_{m+1} .
\end{aligned}
$$

By this lemma we see that the compatibility condition (16) is $H_{n}=0$. Hence we have the following:

THEOREM 1. Suppose that the nth invariant $H_{n-1}$ does not vanish and hence the $(n+1)$ th invariant $H_{n}$ can be defined. Then the linear hyperbolic equation (1) is solved by integrable systems of order $n$ if and only if $H_{n}=0$.

2. An Imschenetsky transformation. Given two functions $M$ and $N$ of $(x, y, z, q)$ take a solution $h$ of $\partial h / \partial z-M(\partial h / \partial q)=0$ satisfying $\partial h / \partial q \neq 0$, and put $k=\partial h / \partial x$ $-N(\partial h / \partial q)$. Then we have

$$
H=\frac{\partial M}{\partial x}-N \frac{\partial M}{\partial q}-\frac{\partial N}{\partial z}+M \frac{\partial N}{\partial q}=-\frac{\partial(h, k)}{\partial(z, q)} /\left(\frac{\partial h}{\partial q}\right)^{2} .
$$

Hence the four functions $x, y, h, k$ are functionally independent if and only if $H \neq 0$. Suppose $H \neq 0$, and solve

$$
x_{1}=x, \quad y_{1}=y, \quad z_{1}=h(x, y, z, q), \quad p_{1}=k(x, y, z, q) ;
$$


conversely,

$$
z=h_{1}\left(x_{1}, y_{1}, z_{1}, p_{1}\right), \quad q=k_{1}\left(x_{1}, y_{1}, z_{1}, p_{1}\right) .
$$

Then we obtain the following identities:

$$
\begin{aligned}
\frac{\partial h}{\partial q} & =-\frac{\partial h_{1}}{\partial p_{1}} \frac{\partial(h, k)}{\partial(z, q)}, \\
\frac{\partial h}{\partial z} / \frac{\partial h}{\partial q} & =-\frac{\partial k_{1}}{\partial p_{1}} / \frac{\partial h_{1}}{\partial p_{1}}, \\
\left(\frac{\partial h}{\partial x}-k\right) / \frac{\partial h}{\partial q} & =\frac{\partial k_{1}}{\partial p_{1}} \frac{d h_{1}}{d x_{1}} / \frac{\partial h_{1}}{\partial p_{1}}-\frac{d k_{1}}{d x_{1}}, \\
-\frac{\partial k}{\partial q} / \frac{\partial h}{\partial q} & =\frac{\partial h_{1}}{\partial z_{1}} / \frac{\partial h_{1}}{\partial p_{1}}, \\
\frac{\partial k}{\partial q} \frac{d h}{d y} / \frac{\partial h}{\partial q}-\frac{d k}{d y} & =\left(\frac{\partial h_{1}}{\partial y_{1}}-k_{1}\right) / \frac{\partial h_{1}}{\partial p_{1}}
\end{aligned}
$$

Hence an equation of the form

$$
s_{1}+M_{1}\left(x_{1}, y_{1}, z_{1}, p_{1}\right) q_{1}+N_{1}\left(x_{1}, y_{1}, z_{1}, p_{1}\right)=0
$$

is the transformed equation of an Imschenetsky transformation (7) if and only if its first invariant $K_{1}=\partial M_{1} / \partial y_{1}-N_{1}\left(\partial M_{1} / \partial p_{1}\right)-\partial N_{1} / \partial z_{1}+M_{1}\left(\partial N_{1} / \partial p_{1}\right)$ with respect to the other characteristic does not vanish. In this case we have $H=K_{1}$.

Let us define the second invariants $H_{1 i}, 1 \leqq i \leqq 4$, of equation (11) of Imschenetsky type by

where

$$
\begin{aligned}
& H_{11}=Z_{1} \frac{\partial \log H}{\partial q}-\frac{\partial M}{\partial q} \frac{\partial \log H}{\partial q}+\frac{\partial^{2} M}{\partial q^{2}}=\frac{\partial L_{1}}{\partial q} \\
& H_{12}=X_{1} \frac{\partial \log H}{\partial q}-\frac{\partial N}{\partial q} \frac{\partial \log H}{\partial q}+\frac{\partial^{2} N}{\partial q^{2}}=\frac{\partial L_{2}}{\partial q} \\
& H_{13}=Z_{1} Q-m_{1} \frac{\partial \log H}{\partial q}+\frac{\partial m_{1}}{\partial q}=\frac{d L_{1}}{d y}-M L_{1}-Z_{1} M \\
& H_{14}=X_{1} Q-n_{1} \frac{\partial \log H}{\partial q}+\frac{\partial n_{1}}{\partial q}=\frac{d L_{2}}{d y}-N L_{1}-Z_{1} N-2 H
\end{aligned}
$$

$$
\begin{gathered}
X_{1}=\partial / \partial x-N \partial / \partial q, \quad Z_{1}=\partial / \partial z-M \partial / \partial q, \\
Q=d(\log H) / d y-2 M, \quad m_{1}=d M / d y-M^{2}, \quad n_{1}=d N / d y-M N, \\
L_{1}=Z_{1} \log H+\partial M / \partial q, \quad L_{2}=X_{1} \log H+\partial N / \partial q .
\end{gathered}
$$

Proposition 2. Equation (11) of Imschenetsky type is solved by integrable systems of the first order if and only if $H_{1 i}=0,1 \leqq i \leqq 4$.

Proof. In our case the system (12) is equivalent to the system of the equations

$$
Z_{1} u+(\partial M / \partial q) u+m_{1}=0, \quad X_{1} u+(\partial N / \partial q) u+n_{1}=0
$$


with independent variables $x, y, z, q$. The compatibility condition is

$$
\partial u / \partial q-(\partial(\log H) / \partial q) u-Q=0 .
$$

Hence the rank is one if and only if $H_{1 i}=0,1 \leqq i \leqq 4$.

Proposition 3. Equation (11) of Imschenetsky type is of Laplace type if and only if $H_{11}=H_{13}=0$.

Proof. Operating successively $H(\partial h / \partial q)\left(\partial / \partial p_{1}\right)=Z_{1}$ to the coefficients

$$
M_{1}=-\frac{\partial k}{\partial q} / \frac{\partial h}{\partial q}, \quad N_{1}=-\frac{d k}{d y}+\frac{\partial k}{\partial q} \frac{d h}{d y} / \frac{\partial h}{\partial q},
$$

we have

$$
-\frac{\partial M_{1}}{\partial p_{1}}=\left\{\frac{\partial}{\partial q} \log \left(\frac{\partial h}{\partial q} H\right)\right\} / \frac{\partial h}{\partial q}, \quad-H\left(\frac{\partial h}{\partial q}\right)^{2} \frac{\partial^{2} M_{1}}{\partial p_{1}^{2}}=H_{11}
$$

and

$$
\begin{aligned}
-\frac{\partial N_{1}}{\partial p_{1}} & =Q+\left[\frac{\partial d h}{\partial q d y}-\frac{d h}{d y}\left\{\frac{\partial}{\partial q} \log \left(H \frac{\partial h}{\partial q}\right)\right\}\right] / \frac{\partial h}{\partial q}, \\
-H \frac{\partial h}{\partial q} \frac{\partial^{2} N_{1}}{\partial p_{1}^{2}} & =H_{13}-\frac{d h}{d y} H_{11} / \frac{\partial h}{\partial q} .
\end{aligned}
$$

Hence $M_{1}$ and $N_{1}$ are linear with respect to $p_{1}$ if and only if $H_{11}=H_{13}=0$.

COROLlary. Equation (11) of Imschenetsky type is of Laplace type if it is solved by integrable systems of the first order.

Suppose that equation (11) is of Laplace type. Then the transformed equation has the form

$$
s_{1}-\left(\gamma p_{1} q_{1}+\alpha p_{1}+\delta q_{1}+\beta\right)=0 .
$$

These coefficients are given by

$$
\begin{gathered}
\gamma=\left(\frac{\partial \log H}{\partial q}+\frac{\partial^{2} h}{\partial q^{2}} / \frac{\partial h}{\partial q}\right) / \frac{\partial h}{\partial q}, \quad \alpha=Q+\frac{\partial d h}{\partial q d y} / \frac{\partial h}{\partial q}-\frac{d h}{d y} \gamma, \\
\delta=\frac{\partial k}{\partial q} / \frac{\partial h}{\partial q}-k \gamma, \quad \beta=\frac{d k}{d y}-\frac{\partial k}{\partial q} \frac{d h}{d y} / \frac{\partial h}{\partial q}-k \alpha .
\end{gathered}
$$

The equation (17) is Monge-integrable if and only if each of $A=\partial \delta / \partial z_{1}-\partial \gamma / \partial x_{1}$, $B=\partial \beta / \partial z_{1}-\partial \alpha / \partial x_{1}+\alpha \delta-\beta \gamma$ vanishes, since the first invariant is $A q_{1}+B$. These invariants are given by

$$
A=-H_{12} / \frac{\partial h}{\partial q}, \quad B=-H_{14}+\frac{d h}{d y} H_{12} / \frac{\partial h}{\partial q} .
$$

Apply Proposition 2 to the equation (17). Then we have the following: 
Proposition 4. Suppose $A \neq 0$ or $B \neq 0$. Then equation (17) is solved by integrable systems of the first order if and only if each of the following invariants $I_{j}, 1 \leqq j \leqq 10$, vanishes:

$$
\begin{aligned}
I_{1}= & B \frac{\partial A}{\partial z_{1}}-A \frac{\partial B}{\partial z_{1}}-\alpha A^{2}+\gamma A B \\
I_{2}= & B \frac{\partial A}{\partial x_{1}}-A \frac{\partial B}{\partial x_{1}}-\beta A^{2}+\delta A B \\
I_{3}= & A \frac{\partial^{2} A}{\partial z_{1}^{2}}-\left(\frac{\partial A}{\partial z_{1}}\right)^{2}+\gamma A \frac{\partial A}{\partial z_{1}}+\left(\frac{\partial \gamma}{\partial z_{1}}+\gamma^{2}\right) A^{2} \\
I_{4}= & B \frac{\partial^{2} B}{\partial z_{1}^{2}}-\left(\frac{\partial B}{\partial z_{1}}\right)^{2}+\gamma B \frac{\partial B}{\partial z_{1}}+\frac{\partial \alpha}{\partial z_{1}} A B+\alpha^{2} A^{2} \\
I_{5}= & A \frac{\partial^{2} A}{\partial y_{1} \partial z_{1}}-\frac{\partial A}{\partial y_{1}} \frac{\partial A}{\partial z_{1}}+\alpha A \frac{\partial A}{\partial z_{1}}+\left(\frac{\partial \alpha}{\partial z_{1}}+\alpha \gamma\right) A^{2} \\
I_{6}= & B \frac{\partial^{2} B}{\partial y_{1} \partial z_{1}}-\frac{\partial B}{\partial y_{1}} \frac{\partial B}{\partial z_{1}}+\alpha B \frac{\partial B}{\partial z_{1}}+\alpha\left(B \frac{\partial A}{\partial y_{1}}-A \frac{\partial B}{\partial y_{1}}\right) \\
& +\left(\frac{\partial \alpha}{\partial y_{1}}+\alpha^{2}\right) A B+\left(\frac{\partial \alpha}{\partial z_{1}}-\frac{\partial \gamma}{\partial y_{1}}\right) B^{2}, \\
I_{7}= & A \frac{\partial^{2} A}{\partial x_{1} \partial z_{1}}-\frac{\partial A}{\partial x_{1}} \frac{\partial A}{\partial z_{1}}+\delta A \frac{\partial A}{\partial z_{1}}+\left(\frac{\partial \delta}{\partial z_{1}}+\gamma \delta-2 A\right) A^{2} \\
I_{8}= & B \frac{\partial^{2} B}{\partial x_{1} \partial z_{1}}-\frac{\partial B}{\partial x_{1}} \frac{\partial B}{\partial z_{1}}+\delta B \frac{\partial B}{\partial z_{1}}+\alpha \beta A^{2}-A B^{2}+\frac{\partial \alpha}{\partial x_{1}} A B \\
I_{9}= & A \frac{\partial^{2} A}{\partial x_{1} \partial y_{1}}-\frac{\partial A}{\partial x_{1}} \frac{\partial A}{\partial y_{1}}+\beta A \frac{\partial A}{\partial z_{1}}+\left(\frac{\partial \beta}{\partial z_{1}}+\alpha \delta-2 B\right) A^{2} \\
& +\left(\frac{\partial \beta}{\partial y_{1}}+\alpha \beta\right) A B+\left(\frac{\partial \alpha}{\partial x_{1}}-\frac{\partial \delta}{\partial y_{1}}\right) B^{2}-B^{3} . \\
I_{10}= & B \frac{\partial^{2} B}{\partial x_{1} \partial y_{1}}-\frac{\partial B}{\partial x_{1}} \frac{\partial B}{\partial y_{1}}+\beta B \frac{\partial B}{\partial z_{1}}+\beta\left(B \frac{\partial A}{\partial y_{1}}-A \frac{\partial B}{\partial y_{1}}\right) \\
I_{1} &
\end{aligned}
$$

Proof. Since we get

$$
\begin{aligned}
& L_{1}=\left(\left(\partial A / \partial z_{1}\right) q_{1}+\partial B / \partial z_{1}+\alpha A-\gamma B\right) /\left(A q_{1}+B\right), \\
& L_{2}=\left(\left(\partial A / \partial x_{1}\right) q_{1}+\partial B / \partial x_{1}+\beta A-\delta B\right) /\left(A q_{1}+B\right),
\end{aligned}
$$

the identities $H_{11}=H_{12}=0$ hold if and only if $I_{1}=I_{2}=0$. Suppose that $I_{1}=I_{2}=0$, and $A \neq 0$. Then $H_{13}=H_{14}=0$ if and only if $I_{3}=I_{5}=I_{7}=I_{9}=0$. In this case the identities $I_{4}=I_{6}=I_{8}=I_{10}=0$ are satisfied. Suppose that $I_{1}=I_{2}=0$, and $B \neq 0$. Then $H_{13}=H_{14}=0$ if and only if $I_{4}=I_{6}=I_{8}=I_{10}=0$. In this case the identities $I_{3}=I_{5}$ $=I_{7}=I_{9}=0$ are satisfied.

REMARK. Identity (18) gives another proof of the following theorem obtained in the previous note [2]:

An equation of Laplace type is solved by integrable systems of the first order if and only if the transformed equation is Monge-integrable. 
3. Integration of an equation of Laplace type. Let us solve equation (11) of Laplace type by integrable systems of the second order. It is possible if and only if the system of the equations

$$
\begin{gathered}
Z_{2} u+(\partial M / \partial q) u+M_{2}=0 \\
X_{2} u+(\partial N / \partial q) u+N_{2}=0
\end{gathered}
$$

with independent variables $x, y, z, q, t\left(=q_{2}\right)$ has its rank greater than zero. Here

$$
\begin{aligned}
Z_{2} & =\partial / \partial z-M(\partial / \partial q)-\left(m_{1}+(\partial M / \partial q) t\right)(\partial / \partial t), \\
X_{2} & =\partial / \partial x-N(\partial / \partial q)-\left(n_{1}+(\partial N / \partial q) t\right)(\partial / \partial t), \\
M_{2} & =\left(d m_{1} / d y-M m_{1}\right)+\left(2\left(\partial m_{1} / \partial q\right)-Z_{1} M\right) t+\left(\partial^{2} M / \partial q^{2}\right) t^{2} \\
N_{2} & =\left(d n_{1} / d y-N m_{1}\right)+\left(2\left(\partial n_{1} / \partial q\right)-Z_{1} N\right) t+\left(\partial^{2} N / \partial q^{2}\right) t^{2}
\end{aligned}
$$

The compatibility condition between equations (19) and (20) is

$$
Y_{2} u-(\partial(\log H) / \partial q) u-Q_{2}=0 \text {, }
$$

where

$$
\begin{aligned}
Y_{2}= & \frac{\partial}{\partial q}+\left(Q+t \frac{\partial \log H}{\partial q}\right) \frac{\partial}{\partial t}, \\
Q_{2}= & \left(Q+t \frac{\partial \log H}{\partial q}\right)^{2}+\left(\frac{d}{d y}+t \frac{\partial}{\partial q}\right)\left(Q+t \frac{\partial \log H}{\partial q}\right) \\
& +M\left(Q+t \frac{\partial \log H}{\partial q}\right)-\left(m_{1}+\frac{\partial M}{\partial q} t\right),
\end{aligned}
$$

since $\left[Z_{2}, X_{2}\right]=H Y_{2}$. The compatibility condition between (20) and (21) is

$$
\left(T+T_{1} t\right)(\partial u / \partial t)-T_{1} u-Q_{3}=0
$$

where $T=H_{14}, T_{1}=H_{12}$,

$$
Q_{3}=2\left(Q+t \frac{\partial \log H}{\partial q}\right)\left(T+T_{1} t\right)+\left(\frac{d}{d y}+t \frac{\partial}{\partial q}\right)\left(T+T_{1} t\right)+M\left(T+T_{1} t\right),
$$

since $\left[X_{2}, Y_{2}\right]=(\partial N / \partial q) Y_{2}+\left(T+T_{1} t\right)(\partial / \partial t)$. The compatibility condition between (19) and (21) is $Y_{2} M_{2}+Z_{2} Q_{2}-(\partial(\log H) / \partial q) M_{2}=0$, since $\left[Z_{2}, Y_{2}\right]=(\partial M / \partial q) Y_{2}$. This condition is identically satisfied by

$$
Z_{2}\left(Q+t \frac{\partial \log H}{\partial q}\right)=-\left(\frac{\partial m_{1}}{\partial q}+\frac{\partial^{2} M}{\partial q^{2}} t\right)
$$

Also the compatibility condition between (19) and (22) is satisfied identically by $Z_{1} T=m_{1} T_{1}, Z_{1} T_{1}=(\partial M / \partial q) T_{1}, Z_{2}\left(T+T_{1} t\right)=0$. Hence the rank is two if and only if $H_{12}=H_{14}=0$.

THEOREM 2. Equation (11) of Laplace type is solved by integrable systems of the second order if and only if the transformed equation (17) is solved by integrable systems of the first order. 
Proof. By (18), $H_{12}=H_{14}=0$ if and only if $A=B=0$. In this case the transformed equation (17) is Monge-integrable and it is solved by integrable systems of the first order. Hence we may assume that $H_{12} \neq 0$ or $H_{14} \neq 0$. The compatibility condition between (21) and (22) is

$$
\begin{aligned}
& \left(T_{1} /\left(T+T_{1} t\right)\right)(\partial(\log H) / \partial q)+Y_{2}\left(T_{1} /\left(T+T_{1} t\right)\right)=0 \\
& \partial Q_{2} / \partial t-\left(T_{1} /\left(T+T_{1} t\right)\right) Q_{2}-Y_{2}\left(Q_{3} /\left(T+T_{1} t\right)\right)=0
\end{aligned}
$$

Let $F$ be the left-hand member of (23) multiplied by $\left(T+T_{1} t\right)^{2}$ :

$$
F=T T_{1}(\partial(\log H) / \partial q)+T\left(\partial T_{1} / \partial q\right)-T_{1}(\partial T / \partial q)-T_{1}^{2} Q
$$

Then we have the identity

$$
F=(\partial h / \partial q)^{2} I_{1}
$$

Express the left-hand member of (24) multiplied by $\left(T+T_{1} t\right)^{2}$ in the polynomial of $t, F_{0}+F_{1} t+F_{2} t^{2}+F_{3} t^{3}$. These coefficients are given by

$$
\begin{aligned}
F_{3}= & -T_{1} \frac{\partial^{2} T_{1}}{\partial q^{2}}-T_{1} \frac{\partial T_{1}}{\partial q} \frac{\partial \log H}{\partial q}+\left(\frac{\partial T_{1}}{\partial q}\right)^{2}-T_{1}^{2}\left\{\left(\frac{\partial \log H}{\partial q}\right)^{2}+\frac{\partial^{2} \log H}{\partial q^{2}}\right\} \\
F_{2}= & \frac{\partial F}{\partial q}+F \frac{\partial \log H}{\partial q}+2 \frac{T}{T_{1}} F_{3}-\frac{2}{T_{1}} \frac{\partial T_{1}}{\partial q} F+F_{2}^{\prime} \\
F_{2}^{\prime}= & -T_{1} \frac{\partial d T_{1}}{\partial q d y}-T_{1} \frac{\partial T_{1}}{\partial q} Q+\frac{\partial T_{1}}{\partial q} \frac{d T_{1}}{d y}-T_{1}^{2}\left(\frac{\partial Q}{\partial q}+\frac{\partial M}{\partial q}+Q \frac{\partial \log H}{\partial q}\right) \\
F_{1}= & \left(\frac{T}{T_{1}}\right)^{2} F_{3}+2 \frac{T}{T_{1}} F_{2}^{\prime}+\frac{T}{T_{1}} \frac{\partial F}{\partial q}+\frac{d F}{d y} \\
& +\left(-2 \frac{d T_{1}}{d y}-\frac{1}{T_{1}} \frac{\partial T}{\partial q}-\frac{T}{T_{1}^{2}} \frac{\partial T_{1}}{\partial q}+\frac{T}{T_{1}} \frac{\partial \log H}{\partial q}+Q+M\right) F \\
F_{0}= & \left(\frac{T}{T_{1}}\right)^{2} F_{2}^{\prime}+\frac{T}{T_{1}} \frac{d F}{d y}+\left\{-\frac{1}{T_{1}} \frac{d T}{d y}-\frac{T}{T_{1}^{2}} \frac{d T_{1}}{d y}+(Q+M) \frac{T}{T_{1}}\right\} F
\end{aligned}
$$

Here we assumed that $T_{1} \neq 0$. If $T_{1}=0$, then we have $F_{3}=F_{2}=0$ and

$$
\begin{aligned}
& F_{1}=-T\left(\frac{\partial^{2} T}{\partial q^{2}}+\frac{\partial T}{\partial q} \frac{\partial \log H}{\partial q}\right)+\left(\frac{\partial T}{\partial q}\right)^{2} \\
& F_{0}=-T \frac{\partial d T}{\partial q d y}+\frac{\partial T}{\partial q} \frac{d T}{d y}-T \frac{\partial T}{\partial q} Q+T^{2}\left(\frac{d \partial \log H}{d y \partial q}+\frac{\partial \log H}{\partial q} M-\frac{\partial Q}{\partial q}-2 \frac{\partial M}{\partial q}\right)
\end{aligned}
$$

Suppose $T_{1} \neq 0$. Then we obtain

$$
F_{3}=-(\partial h / \partial q)^{4} I_{3}, \quad F_{2}^{\prime}=-(\partial h / \partial q)^{3}\left(I_{5}+(d h / d y) I_{3}\right) .
$$

If $T_{1}=0$, then we have

$$
F_{1}=-(\partial h / \partial q)^{2} I_{4}, \quad F_{0}=-(\partial h / \partial q)\left(I_{6}+(d h / d y) I_{4}\right)
$$


The compatibility condition between (20) and (22) is

$$
\begin{aligned}
\frac{T_{1}}{T+T_{1} t} \frac{\partial N}{\partial q}-X_{2}\left(\frac{T_{1}}{T+T_{1} t}\right) & =0 \\
\frac{\partial N_{2}}{\partial t}-\frac{T_{1}}{T+T_{1} t} N_{2}+X_{2}\left(\frac{Q_{3}}{T+T_{1} t}\right) & =0 .
\end{aligned}
$$

These conditions are written in the following forms:

$$
T T_{1} R_{1}-T_{1}(\partial T / \partial x)+T\left(\partial T_{1} / \partial x\right)-T_{1}^{2} R=N F
$$

$$
\frac{\partial}{\partial t}\left(N Q_{2}-N_{2}\right)-\frac{T_{1}}{T+T_{1} t}\left(N Q_{2}-N_{2}\right)-X\left(\frac{T_{1}}{T+T_{1} t}\right)=\frac{-N}{\left(T+T_{1} t\right)^{2}} \sum_{j=0}^{3} F_{j} t^{j}
$$

where $R=N Q-n_{1}, R_{1}=N(\partial(\log H) / \partial q)-\partial N / \partial q, X=X_{2}+N Y_{2}=\partial / \partial x+\left(R+R_{1} t\right)$ $\cdot(\partial / \partial t)$. By the identity $\left[X-N Y_{2}, Y_{2}\right]=(\partial N / \partial q) Y_{2}+\left(T+T_{1} t\right)(\partial / \partial t)$, we get

$$
\partial Q / \partial x+R(\partial(\log H) / \partial q)-\partial R / \partial q-R_{1} Q=T, \quad \partial^{2}(\log H) / \partial x \partial q-\partial R_{1} / \partial q=T_{1},
$$

and

$$
\frac{\partial R}{\partial q}-\frac{d R_{1}}{d y}-R \frac{\partial \log H}{\partial q}+R_{1}(Q+M)+N\left(\frac{\partial M}{\partial q}-\frac{\partial \log H}{\partial z}\right)+\frac{\partial N}{\partial z}=0
$$

Let $E$ be the left-hand member of (28). Then we obtain

$$
E=(\partial h / \partial q)\left(I_{2}+(\partial h / \partial x) I_{1}\right)
$$

Express the left-hand member of (29) multiplied by $\left(T+T_{1} t\right)^{2}$ in the polynomial of $t, E_{0}+E_{1} t+E_{2} t^{2}+E_{3} t^{3}$. These coefficients are given by

$$
\begin{aligned}
E_{3}= & -T_{1} \frac{\partial^{2} T_{1}}{\partial x \partial q}+\frac{\partial T_{1}}{\partial x} \frac{\partial T_{1}}{\partial q}-T_{1} \frac{\partial T_{1}}{\partial q} R_{1}+T_{1}^{2}\left(\frac{\partial R_{1}}{\partial q}-R_{1} \frac{\partial \log H}{\partial q}-2 \frac{\partial^{2} \log H}{\partial x \partial q}\right) \\
E_{2}= & 2 \frac{T}{T_{1}} E_{3}+\frac{\partial E}{\partial q}-\frac{2}{T_{1}} \frac{\partial T_{1}}{\partial q} E+R_{1} F+E_{2}^{\prime} \\
E_{2}^{\prime}= & -T_{1} \frac{\partial d T_{1}}{\partial x d y}-T_{1} \frac{\partial T_{1}}{\partial q} R+\frac{\partial T_{1}}{\partial x} \frac{d T_{1}}{d y} \\
& -T_{1}^{2}\left(2 \frac{\partial Q}{\partial x}+\frac{\partial M}{\partial x}+2 R \frac{\partial \log H}{\partial q}-\frac{\partial R}{\partial q}-R_{1} Q\right) \\
E_{1}= & \left(\frac{T}{T_{1}}\right)^{2} E_{3}+2 \frac{T}{T_{1}} E_{2}^{\prime}+\frac{d E}{d y}+\left(\frac{-2}{T_{1}} \frac{d T_{1}}{d y}+Q\right) E \\
& +\frac{T}{T_{1}} \frac{\partial F}{\partial x}+\left(-\frac{1}{T_{1}} \frac{\partial T}{\partial x}+\frac{T}{T_{1}} R_{1}-\frac{T}{T_{1}^{2}} \frac{\partial T_{1}}{\partial x}\right) F \\
= & \left(\frac{T}{T_{1}}\right)^{2} E_{2}^{\prime}+\frac{T}{T_{1}} \frac{d E}{d y}-\frac{1}{T_{1}}\left(\frac{d T}{d y}+\frac{T}{T_{1}} \frac{d T_{1}}{d y}\right) E+\frac{T}{T_{1}} R F
\end{aligned}
$$


Here we assumed that $T_{1} \neq 0$. If $T_{1}=0$, then we have $E_{3}=E_{2}=0$ and

$$
\begin{aligned}
& E_{1}=-T \frac{\partial^{2} T}{\partial x \partial q}+\frac{\partial T}{\partial x} \frac{\partial T}{\partial q}-T \frac{\partial T}{\partial q} R_{1} \\
& E_{0}=-T \frac{\partial d T}{\partial x d y}+\frac{\partial T}{\partial x} \frac{d T}{d y}-T \frac{\partial T}{\partial q} R-T^{2}\left\{2 T+H+N\left(\frac{\partial \log H}{\partial z}-M \frac{\partial \log H}{\partial q}\right)\right\} .
\end{aligned}
$$

Suppose $T_{1} \neq 0$. Then we obtain

$$
\begin{aligned}
& E_{3}=-(\partial h / \partial q)^{3}\left(I_{7}+(\partial h / \partial x) I_{3}\right) \\
& E_{2}^{\prime}=-(\partial h / \partial q)^{2}\left(I_{9}+(d h / d y) I_{7}+(\partial h / \partial x) I_{5}+(\partial h / \partial x)(d h / d y) I_{3}\right)
\end{aligned}
$$

If $T_{1}=0$, then we have

$$
\begin{aligned}
& E_{1}=-(\partial h / \partial q)\left(I_{8}+(\partial h / \partial x) I_{4}\right) \\
& E_{0}=-\left(I_{10}+(d h / d y) I_{8}+(\partial h / \partial x) I_{6}+(d h / d y)(\partial h / \partial x) I_{4}\right) .
\end{aligned}
$$

Hence the given equation of Laplace type is solved by integrable systems of the second order if and only if $E=E_{i}=F_{j}=F=0,0 \leqq i, j \leqq 3$. And it is possible if and only if $I_{k}=0,1 \leqq k \leqq 10$.

COROllary. An equation of Laplace type is transformed to a Monge-integrable equation by applying once or twice the associated Imschenetsky transformation if and only if it is solved by integrable systems of the second order.

EXAMPLE. Take $h=\log q-\log w^{\prime}, k=-w+z-\left(w / w^{\prime}\right) q+w^{\prime}(z / q)$, where $w$ is a function of $x, y$ satisfying $\partial^{2}(\log w) / \partial x \partial y=0, w^{\prime}=\partial w / \partial y \neq 0$. Then the equation

$$
s-w^{\prime} z+\left(w-\partial\left(\log w^{\prime}\right) / \partial x\right) q-z q+\left(w / w^{\prime}\right) q^{2}=0
$$

is transformed to

$$
s_{1}+\left(1+\exp \left(z_{1}\right)\right)^{-1} p_{1} q_{1}+w\left(\exp \left(z_{1}\right)+1\right) q_{1}=0 .
$$

The transformed and original equations are solved by integrable systems of the first and second order respectively. Here $A=-w \exp \left(z_{1}\right), B=0$. Hence the transformed equation is not Monge-integrable.

REMARK. Clairin [1] obtained the following result: Consider three equations

$$
s_{i}-\left(\gamma_{i} p_{i} q_{i}+\alpha_{i} p_{i}+\delta_{i} q_{i}+\beta_{i}\right)=0, \quad 1 \leqq i \leqq 3,
$$

where $\alpha_{i}, \beta_{i}, \gamma_{i}, \delta_{i}$ are functions of $x_{i}, y_{i}, z_{i}, 1 \leqq i \leqq 3$. Suppose equation (33.i) is of Imschenetsky type, and equation $(33 . i+1)$ is the transformed equation of (33.i), $1 \leqq i \leqq 2$. Then equation (33.2) is equivalent to one of the following equations:

$$
\begin{aligned}
& s+a p+b q+c z=0, \quad s-e^{z}+a=0 \\
& s-a e^{-z} p+e^{z} q+(\partial a / \partial x) e^{-z}+b=0
\end{aligned}
$$

where $a, b, c$ are functions of $x, y$. 
1. J. Clairin, Sur la transformation d'Imschenetsky, Bull. Soc. Math. France 41 (1913), 206-228.

2. M. Matsuda, Two methods of integrating Monge-Amperre's equations, Trans. Amer. Math. Soc. 150 (1970), 327-343. MR 41 \#5754.

Department of Mathematics, Osaka University, Toyonaka, Osaka 560, JaPan 